\title{
Discrete element modelling (DEM) input parameters: understanding their impact on model predictions using statistical analysis
}

\author{
Z. Yan $^{1}$ - S. K. Wilkinson ${ }^{1}$ - E. H. Stitt ${ }^{1}$ - M. Marigo ${ }^{1}$
}

Received: 9 December 2014 / Revised: 18 June 2015 / Accepted: 23 June 2015 / Published online: 14 July 2015

(C) OWZ 2015

\begin{abstract}
Selection or calibration of particle property input parameters is one of the key problematic aspects for the implementation of the discrete element method (DEM). In the current study, a parametric multi-level sensitivity method is employed to understand the impact of the DEM input particle properties on the bulk responses for a given simple system: discharge of particles from a flat bottom cylindrical container onto a plate. In this case study, particle properties, such as Young's modulus, friction parameters and coefficient of restitution were systematically changed in order to assess their effect on material repose angles and particle flow rate (FR). It was shown that inter-particle static friction plays a primary role in determining both final angle of repose and FR, followed by the role of inter-particle rolling friction coefficient. The particle restitution coefficient and Young's modulus were found to have insignificant impacts and were strongly cross correlated. The proposed approach provides a systematic method that can be used to show the importance of specific DEM input parameters for a given system and then potentially facilitates their selection or calibration. It is concluded that shortening the process for input parameters selection and calibration can help in the implementation of DEM.
\end{abstract}

Keywords Discrete element method . DEM input parameters - DEM calibration - Parameter sensitivity analysis

Electronic supplementary material The online version of this article (doi:10.1007/s40571-015-0056-5) contains supplementary material, which is available to authorized users.

\section{S. K. Wilkinson}

Sam.Wilkinson@matthey.com

1 Johnson Matthey Technology Centre, P.O. Box 1, Belasis Avenue, Billingham, Cleveland TS23 1LB, UK

\section{Introduction}

In recent years there has been an increase in the application of discrete element method (DEM), originated by Cundall and Strack [1], to study granular materials. The importance of DEM has been demonstrated in different fields spanning chemical engineering, pharmaceuticals, powder metallurgy, agriculture and many others. DEM simulations have been extensively applied to diverse problems in granular processes such as packing of particles [2-4], flow from a hopper [5-7], die filling [8,9], fragmentation of agglomerates [10,11], bulk compression [12-15], flow in a screw conveyor $[16,17]$ and powder mixing [18-21].

DEM is based on the description of the microscopic contacts between a number of discrete particles. The reliability of a DEM model depends on the accuracy of the contact model and the particle property parameters chosen. The choice of DEM input parameters is still a key limitation and an aspect of controversy for the effective application of this modelling tool. Often DEM contact models include the definition of input parameters that can only be measured with difficulties. Measuring single particle properties, especially at a small scale, can be very challenging, costly and time consuming. Furthermore it can be difficult to relate these measurements to models implemented in DEM. A few attempts have been made to measure the Young's modulus of single particles [22,23], the yield stress [24] and interfacial energy using atomic force microscopy $[25,26]$. A common approach is to choose the DEM input parameters by calibrating the DEM model against bulk global measurements and then to adjust these input parameters until the outputs match the experimental, usually macroscopic, observations [27]. This can be a very lengthy procedure and does not challenge the lack of physical understanding in the underpinning model parameters, thus presenting some questions of reliability. 
In order to make trustworthy quantitative rather than qualitative predictions, correct input material parameters should be properly selected and their effect should be properly understood. Moreover, another key aspect to be considered is validation of model predictions by experiment $[28,29]$.

Fundamentally, model parameters should act as estimates for physically meaningful values. In this context, it is important to understand which 'assumed' material parameters have the biggest impact on the simulation results. Sensitivity analysis methods, which aim to understand the impact that model parameters have on model responses, play a critical role in this. To achieve this, it is critically important that the parameters are statistically significant, whether 'assumed' or estimated by numerical solver methods. A common approach with estimated parameters is to assess uncertainty in parameter estimates using a $95 \%$ confidence interval. The value of this is influenced by correlation between observations, noise in the data and degrees of freedom in the estimation process [30]. When a confidence interval is greater than the estimated value, the fitting parameter can be seen as indiscriminate from zero and discounted. In DEM studies, this approach can be used when assessing the impact of inputs parameters on bulk model responses; for example the effect of static/rolling friction coefficients and restitution coefficient on angle of repose in a sandpile test [31]. In this reference a power law relationship between angle of repose response and inputs parameters is used but unfortunately the authors do not report statistical confidence intervals of the fitted power terms.

For more systematic and robust approaches, methods can be found in other fields such as reaction kinetics [32]. Parameter sensitivity on a local and global basis can be analysed via the parameter sensitivity matrix, $S$, which comprises the derivatives of all model responses with respect to model parameters:

$S=\frac{\delta \boldsymbol{f}}{\delta \boldsymbol{P}}$

where the model response function, $\boldsymbol{f}$, has size $n \times m$, where $n$ is the number of observations and $m$ is the number of measured outputs at each observation. $\boldsymbol{P}$ is a parameter vector of size $k$ and $S$ is a parameter sensitivity matrix of size $n m \times k$ [33]. Low sensitivity parameters can be systematically identified as low importance parameters in the model by this analysis process $[34,35]$. Cross correlation between parameters, which impedes reliable parameter estimates, is also assessed with these methods [36].

Principal component analysis (PCA) can also be applied to the sensitivity values of model responses with respect to model parameters. PCA provides a ranked series of eigenvalues which contain contributions from the fitting parameters. The most important parameters will have a strong contribution to the largest eigenvalues [37-39]. In DEM, the method was recently applied in the modelling of a continuous convective mixer [40].

Sensitivity analysis of model outputs with respect to model parameters can also be applied using the underpinning mechanistic equations which are solved in DEM simulations (e.g., Hertz-Mindlin equation). Such approaches can demonstrate the importance of model DEM parameters on a local rather than bulk scale. These could ultimately be compared to bulk observations in DEM (e.g., angle of repose) to gauge the importance of model parameters on multiple scales. This approach, to the authors' knowledge, has not been attempted in the literature.

In this paper, a parameter sensitivity analysis methodology to understand the importance of the input parameters and/or their cross-correlation is proposed. The aims for this work are thus:

- Develop empirical sensitivity analysis methodologies which relate choice of input parameter values to 'bulk' model responses such as angles of repose and flow rate (FR).

- Develop mechanistically driven sensitivity analysis methodologies which relate choice of input parameter values to calculated responses using the fundamental equations used in DEM (e.g., Hertz-Mindlin equation outputs).

- Apply approaches to a model system, the sandpile test, to understand which parameters have the greatest impact on model responses. Identify which model responses are key discriminators in this case.

- Evaluate the potential for parameter sensitivity analysis in the understanding of different particle motion mechanics for a given system, in this case the sandpile test.

In this paper, Sects. 2.1 and 2.2 describe the methodologies including the DEM method and parameter sensitivity study approach, respectively. Section 3 presents the background information on the hopper discharge system under study including geometric setup, input parameter ranges and measured outputs. Section 4.1 presents the results from the DEM simulations for the system under study and includes general trends and observations. Section 4.2 uses parameter estimation and sensitivity analysis approaches to understand the importance of the DEM input parameters in the system from both an empirical and mechanistic standpoint. Section 5 is the summary of this research.

\section{Methodologies}

\subsection{Discrete element method (DEM)}

The DEM employed in this work uses the soft-sphere approach originally developed by Cundall and Strack [1]. 
The motions of individual particles are determined by the equations of motion, Eqs. (2) and (3). In this work the forces and torques due to gravity, deformation due to collisions, and static and rolling friction are considered for a particle $i$ in contact with particle $j$ as follows:

$$
\begin{aligned}
\mathrm{m}_{\mathrm{i}} \frac{\mathrm{d} \boldsymbol{v}_{\mathrm{i}}}{\mathrm{dt}} & =\sum\left(\boldsymbol{F}_{\mathrm{ij}}^{\mathrm{n}}+\boldsymbol{F}_{\mathrm{ij}}^{\mathrm{t}}\right)+\mathrm{m}_{\mathrm{i}} \boldsymbol{g}, \\
I_{\mathrm{i}} \frac{\mathrm{d} \omega_{\mathrm{i}}}{\mathrm{dt}} & =\sum\left(\boldsymbol{R}_{\mathrm{i}} \times \boldsymbol{F}_{\mathrm{ij}}^{\mathrm{t}}-\boldsymbol{\tau}_{\mathrm{ij}}^{\mathrm{r}}\right),
\end{aligned}
$$

where $m_{i}, \boldsymbol{g}, I_{i}, \boldsymbol{v}_{i}$ and $\omega_{i}$ are, respectively, the mass, gravity vector, moment of inertia, translational velocity and rotational velocity of particle $i . \boldsymbol{F}_{i j}^{n}$ and $\boldsymbol{F}_{i j}^{t}$ are the normal and the tangential forces due to interaction between particle $i$ and $j$ at the current time-step as depicted in Fig. $1 . \boldsymbol{R}_{i}$ is the vector between the centre of particle $i$ and the contact point where the force $\boldsymbol{F}_{i j}^{t}$ is applied. $\boldsymbol{\tau}_{i j}^{r}$ is the torque due to rolling friction.

In this work, an adapted Hertz-Mindlin contact model is utilised. The contact force can be broken down into normal and tangential non-linear contact forces, as shown in Eqs. (4) and (5). They consist of two terms. The first term stands for the non-linear elastic Hertz model in the normal direction and the linear elastic Mindlin model in the tangential direction $[41,42]$. In both normal and tangential directions a dissipative term is added to account for energy dissipation during collisions through inelastic deformation and friction. In the collision between two spheres $i$ and $j$ represented in Fig. 1, the normal and tangential forces, $\boldsymbol{F}_{i j}^{n}$ and $\boldsymbol{F}_{i j}^{t}$ are given by:

$\boldsymbol{F}_{\mathrm{ij}}^{\mathrm{n}}=-\frac{4}{3} E^{*} \sqrt{R^{*} \delta_{\mathrm{ij}}^{\mathrm{n}}} \delta_{\mathrm{ij}}^{\mathrm{n}}-2 \sqrt{\frac{5}{6}} \psi \sqrt{\mathrm{C}_{\mathrm{n}} \mathrm{m}^{*}} \boldsymbol{v}_{\mathrm{ij}}^{\mathrm{n}}$

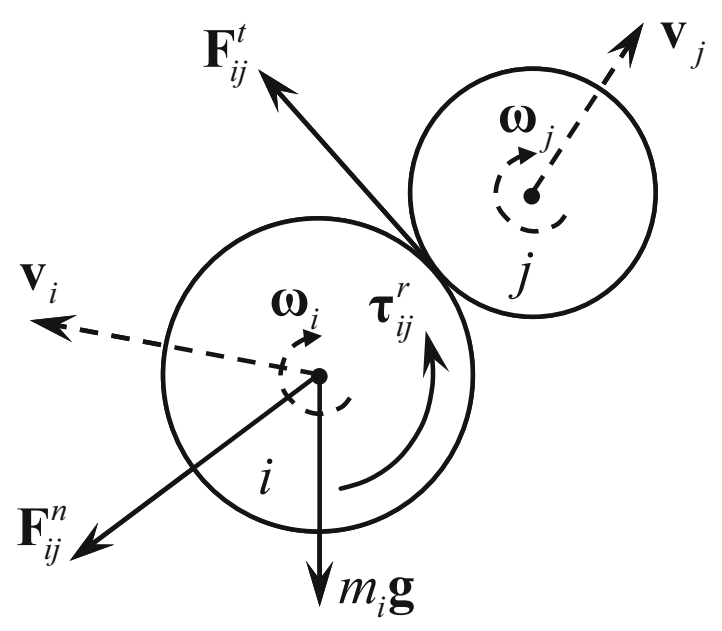

Fig. 1 Schematic representation for the contact between two particles $i$ and $j$
$\boldsymbol{F}_{\mathrm{ij}}^{\mathrm{t}}=-8 G^{*} \sqrt{R^{*} \delta_{\mathrm{ij}}^{\mathrm{n}}} \delta_{\mathrm{ij}}^{\mathrm{t}}-2 \sqrt{\frac{5}{6}} \psi \sqrt{\mathrm{C}_{\mathrm{t}} \mathrm{m}^{*}} \boldsymbol{v}_{\mathrm{ij}}^{\mathrm{t}}$,

where $E^{*}$ is the equivalent Young's modulus of the two colliding particles, defined by $\frac{1}{E^{*}}=\frac{1-v_{i}^{2}}{E_{i}}+\frac{1-v_{j}^{2}}{E_{j}}$, where $v_{\mathrm{i}}$ and $v_{\mathrm{j}}$ are the Poisson's ratios, $R^{*}$ is the equivalent radius, defined by $\frac{1}{R^{*}}=\frac{1}{R_{i}}+\frac{1}{R_{j}}, m^{*}$ is the equivalent mass, defined by $\frac{1}{m^{*}}=\frac{1}{m_{i}}+\frac{1}{m_{j}}, \boldsymbol{v}_{i j}^{n}$ and $\boldsymbol{v}_{i j}^{t}$ are the normal and tangential components of the relative velocity at the contact, $\delta_{i j}^{n}$ is the normal contact overlap, given by $\left|\delta_{i j}^{n}\right|=R_{i}+R_{j}-d_{i j}$, where $d_{i j}$ is the distance of the centre of particles, $\delta_{i j}^{t}$ is tangential contact overlap, given by the integral of the tangential relative velocity through the collision time since the collision starts, i.e., $\left|\delta_{i j}^{t}\right|=\int_{0}^{t}\left|\boldsymbol{v}_{i j}^{t}\right| d t, C_{n}=2 E^{*} \sqrt{R^{*} \delta_{i j}^{n}}$ and $C_{t}=8 G^{*} \sqrt{R^{*} \delta_{\mathrm{ij}}^{\mathrm{n}}}$ are the normal and tangential contact stiffness, where $G^{*}$ is the equivalent shear modulus, defined as $\frac{1}{G^{*}}=\frac{2\left(2-v_{\mathrm{i}}\right)\left(1+v_{\mathrm{i}}\right)}{E_{\mathrm{i}}}+\frac{2\left(2-v_{\mathrm{j}}\right)\left(1+v_{\mathrm{j}}\right)}{E_{\mathrm{j}}}, \psi$ is the damping ratio coefficient which is a function of the coefficient of restitution, $\varepsilon$, given by $\psi=\ln (\varepsilon) / \sqrt{\ln ^{2}(\varepsilon)+\pi^{2}}$.

Sufficient tangential forces will cause particles to slip relative to each other or to other surfaces with which they are in contact. For non-cohesive particles subject to a constant normal force, the extent of slippage under tangential force is determined by:

$\left|\boldsymbol{F}_{\mathrm{ij}}^{\mathrm{t}}\right|<\mu_{\mathrm{s}}\left|\boldsymbol{F}_{\mathrm{ij}}^{\mathrm{n}}\right|$

where $\mu_{s}$ is the static friction coefficient between particles. If Eq. (6) is satisfied, the effect of $\boldsymbol{F}_{i j}^{t}$ is to cause a small relative movement, termed 'microslip' and Eq. (5) is used as tangential force. If Eq. (6) is not satisfied, the slip covers all the area of contact and this can be referred as 'gross sliding' [19]. In this case the tangential force is given by Coulomb's friction law:

$\boldsymbol{F}_{\mathrm{ij}}^{\mathrm{t}}=-\mu_{\mathrm{s}}\left|\boldsymbol{F}_{\mathrm{ij}}^{\mathrm{n}}\right| \frac{\boldsymbol{\delta}_{\mathrm{ij}}^{\mathrm{t}}}{\left|\boldsymbol{\delta}_{\mathrm{ij}}^{\mathrm{t}}\right|}$

In essence, this means that the tangential force acting on the particle will be minimum calculated from Eqs. (5) to (7).

The term $\boldsymbol{\tau}_{i j}^{r}$ in Eq. (3) is added to account for the torque caused by rolling friction. The 'rolling friction' is a term used to define the 'resistance to rolling'. Rolling resistance is often introduced to represent the effects of particle shape on rolling. Different rolling resistance models have been developed and these models have been recently reviewed by Ooi and colleagues [43]. In this work, the elastic-plastic-spring-dashpot (EPSD) rolling resistance model [43] is used to calculate the $\boldsymbol{\tau}_{i j}^{r}$ term. $\boldsymbol{\tau}_{i j, t}^{r}$ is the torque at time $t$ and the incremental torque $\Delta \boldsymbol{\tau}_{i j, t}^{r}$ is calculated from the incremental relative 
rotation between two particles $\Delta \varphi_{i j}^{r}$ and the rolling stiffness $8 G^{*} \sqrt{R^{*} \delta_{i j}^{n}} R^{* 2}$.

$$
\begin{aligned}
& \Delta \boldsymbol{\tau}_{\mathrm{ij}}^{\mathrm{r}}=-8 G^{*} \sqrt{R^{*} \delta_{i j}^{n}} R^{* 2} \Delta \varphi, \\
& \boldsymbol{\tau}_{\mathrm{ij}, \mathrm{t}+\Delta t}^{\mathrm{r}}=\boldsymbol{\tau}_{\mathrm{ij}, \mathrm{t}}^{\mathrm{r}}+\Delta \boldsymbol{\tau}_{\mathrm{ij}}^{\mathrm{r}}, \\
& \left|\boldsymbol{\tau}_{\mathrm{ij}, \mathrm{t}+\Delta \mathrm{t}}^{\mathrm{r}}\right| \leq \mu_{\mathrm{r}} \mathrm{R}^{*}\left|\boldsymbol{F}_{\mathrm{ij}}^{\mathrm{n}}\right|,
\end{aligned}
$$

where $\mu_{r}$ is the rolling friction coefficient. In this EPSD rolling resistance model, torque $\boldsymbol{\tau}_{i j}^{r}$ is similar to the loadingunloading stress-strain curve of an elastic perfectly plastic material. This advanced model includes the rolling back curve which makes it applicable to both one way rolling and cyclic rolling [42]. The EPSD rolling resistance model is implemented and available in the public release of LIGGGHTS source codes.

In this work the time step $\Delta t$ was selected equal to 10 $20 \%$ of the critical time step $T_{R} . T_{R}$ was estimated based on the Rayleigh wave speed of the smallest sphere and was calculated through the equation proposed by Thornton and Randall [44].

$\Delta t<T_{R}=\frac{\pi R \sqrt{\rho / G}}{0.01631 v+0.8766}$,

where $\rho$ is the particle density, $v$ the Poisson's ratio.

\subsection{Parameter sensitivity analysis methods}

In this work, a three-level parameter sensitivity study will be carried out, as shown in Fig. 2. This will allow an understanding of the level of detail required to understand parameter sensitivity in a DEM model and assess consistency of the outcomes between the methods.

In Fig. 2, the complexity is seen to increase from Level 1 through 3 as more detailed input information is required for the sensitivity analysis. The increase in complexity does not

\begin{tabular}{|c|c|c|}
\hline $\begin{array}{l}\quad \text { Level } 1 \\
\text { - Impact of changing } \\
\text { input parameters on } \\
\text { bulk responses of the } \\
\text { system (e.g. particle } \\
\text { flow rate, angle of } \\
\text { repose) } \\
\text { - Parameter estimation } \\
\text { methods }\end{array}$ & \begin{tabular}{|l}
\multicolumn{1}{c}{ Level 2 } \\
- Impact of input \\
parameters \\
sensitivities on bulk \\
responses of the \\
system (e.g. particle \\
flow rate, angles of \\
repose) \\
- Principal component \\
analysis (PCA)
\end{tabular} & \begin{tabular}{l}
\multicolumn{1}{c}{ Level 3 } \\
- Impact on input \\
parameter \\
sensitivities on \\
intrinsic model \\
responses (e.g. \\
contact force, torque) \\
- a priori calculations
\end{tabular} \\
\hline
\end{tabular}
necessarily result in the approach being more time consuming; a topic which is addressed in Sect. 4.2.3.

Fig. 2 Levels of sensitivity analysis utilised in this study
The subsequent Sects. 2.2.1-3 address the methodologies used in the three levels of parameter sensitivity analysis. The specifics of the models used as part of these methodologies are described in the results Sects. 4.2.1-3.

\subsubsection{Level 1 method: parameter estimation}

Parameter estimation was carried out using Athena Visual Studio v14.2 (C) software (Athena Visual Software, Inc., Naperville, IL). All empirical models are algebraic equations in structure and estimation of their parameters can be carried out explicitly:

$y_{i}=f_{i}\left(\xi_{i} ; P_{k}\right)+e_{i}$,

where $y_{i}$ is a model response, $\xi_{i}$ are the process settings (input variables), $P_{k}$ are model parameters and $e_{i}$ is model error, where $i \leq n$. A non-linear least squares method was used to estimate the empirical model parameters. This is owed to the presence of some non-linear parameters (e.g., power indices) within the model. The method is also suitable for single response parameter estimation problems which are used in this study.

\subsubsection{Level 2 method: principal component analysis (PCA)}

PCA was carried out using a program developed in Athena Visual Studio v14.2 () software. The aim of this method is to understand the relationship between bulk model responses and associated parameter sensitivities. This is achieved by a series of transforms of the sensitivity matrix, the output of which establishes the parameters which have the strongest impact on model responses.

In all cases the input is based on the parameter sensitivity matrix, $S$, as defined in Eq. (1). This matrix differs slightly to Eq. (1) however and contains $(k+1)$ columns. The additional $(k+1)$ th column accounts for a bulk model response in the system (such as angle of repose) which is analysed against the sensitivity values.

For this matrix of size $n m \times(k+1)$, it is necessary to autoscale values in each column (e.g., column size $n m$ for each column of total $(k+1))$. This will enable each parameter sensitivity range to be explored on a relative level. Autoscaling is carried out as follows:

$\boldsymbol{S}_{i, \text { scaled }}=\frac{\boldsymbol{S}_{i}-\boldsymbol{S}_{n m, \text { ave }}}{\boldsymbol{S}_{n m, \mathrm{var}}}$,

where $S_{i}$ is a sensitivity value located in column size $n m$ where $i \leq n m, S_{n m, a v e}$ is the average sensitivity value in column $n m, \boldsymbol{S}_{n m, v a r}$ is the variance of all sensitivity values in column $n m$ and $\boldsymbol{S}_{i, \text { scaled }}$ is the scaled sensitivity value located in column size $n m$ where $i \leq n m$. Across $(k+1)$ columns, this 
leads to an auto-scaled sensitivity matrix, $\boldsymbol{S}_{\text {scaled }}$. The autoscaled matrix can subsequently afford a direct understanding of the impact of parameter sensitivities on model responses.

Subsequently, matrix $\boldsymbol{S}_{\text {scaled }}$ is computed into a covariance matrix, $\boldsymbol{Q}$ [45]:

$\boldsymbol{Q}=\boldsymbol{S}_{\text {scaled }}^{T} \boldsymbol{S}_{\text {scaled }}$

where superscript $T$ denotes matrix transpose. $Q$ is then converted into principal components via the following relationships:

$Q=U \Lambda U^{T}$,

$U Q U^{T}=U^{T} U \Lambda U^{T} U=\Lambda$,

where $\boldsymbol{\Lambda}$ denotes a diagonal matrix of principal components (eigenvalues) of size $k$ and $\boldsymbol{U}$ is the corresponding eigenvector matrix with rows corresponding to $k$. On each row in $\boldsymbol{U}$, the eigenvectors correspond to the $k$ parameters in the system. Hence the importance of each parameter as a function of its sensitivity can be identified for each principal component.

\subsubsection{Level 3 method: parameter sensitivity matrix analysis}

This form of analysis begins with the parameter sensitivity matrix, $S$, of size $n m \times k$, as defined in Eq. (1). The values in each column (i.e., for each parameter $1,2, \ldots, k$ ) are then processed and summed into a form known as the norm of the parameter sensitivity matrix [33]:

$\operatorname{norm}(S)_{k}=\sqrt{\sum_{i}\left(P_{k} \frac{\delta \boldsymbol{f}}{\delta P_{k}}\right)^{2}}$,

where $\operatorname{norm}(\boldsymbol{S})_{k}$ is the norm of the parameter sensitivity matrix for parameter $1,2, \ldots, k$ and $i \leq n m$. In effect this method provides a summated representation of the sensitivities for a particular parameter in the dataset. Each sensitivity value is scaled by the value of the parameter itself as a normalisation technique. For a system of $k$ parameters, a vector of $\operatorname{norm}(\boldsymbol{S})_{k}$ values of size $k$ will be generated. The magnitude of each of the values in the vector can then be compared. The smallest $\operatorname{norm}(\boldsymbol{S})_{k}$ corresponds to the least influential parameter in the model.

An extension of parameter sensitivity matrix analysis involves studying cross correlation of parameters; a further measure of over parameterisation in a model. In this analysis, each parameter sensitivity column of size $n m$ will be compared to all other parameters sensitivity columns in the system. This can be calculated for all parametric interactions using the following equation [46]:
$(C C)_{k 1, k 2}=\frac{\left(\boldsymbol{S}^{\boldsymbol{T}} \boldsymbol{S}\right)_{k 1, k 2}^{-1}}{\left[\left(\boldsymbol{S}^{\boldsymbol{T}} \boldsymbol{S}\right)_{k 1, k 1}^{-1} \cdot\left(\boldsymbol{S}^{\boldsymbol{T}} \boldsymbol{S}\right)_{k 2, k 2}^{-1}\right]^{0.5}}$,

where $C C$ denotes cross correlation coefficient, which is always in the range $-1<C C<+1$, superscript $T$ denotes matrix transpose and $k_{1}$ and $k_{2}$ are the two considered parameters, respectively. $C C$ values approaching -1 or +1 suggest a strong cross correlation between model parameters.

\section{DEM simulations}

\subsection{Simulation conditions and input parameters}

In this study, a flat bottom cylindrical hopper $(D=$ $50 \mathrm{~mm}, H=70 \mathrm{~mm}$ ) was chosen as a simple test case in order to describe the multi-level sensitivity method approach implementation of the DEM input parameters. The system was implemented using the open source DEM code LIGGGHTS [47] and was carried out on a four-node high performance cluster using 64 CPUs (Intel Xeon $2.20 \mathrm{GHz}$ ) and MPI parallelization. As depicted in Fig. 3, an orifice $(d=15 \mathrm{~mm})$ was in the bottom centre of the cylinder, at a distance of $70 \mathrm{~mm}$ from a plate. The cylinder was filled with monosized $(R=1 \mathrm{~mm})$ spherical particles (total: $\sim 14,700)$ using a pouring scheme in line with the previous literature $[48,49]$ and with all the simulations starting from the same initial packing condition (packing density $56.7 \%$ ). The orifice lid was quickly opened and particles began to discharge under gravity forming a pile of material on the plate beneath.

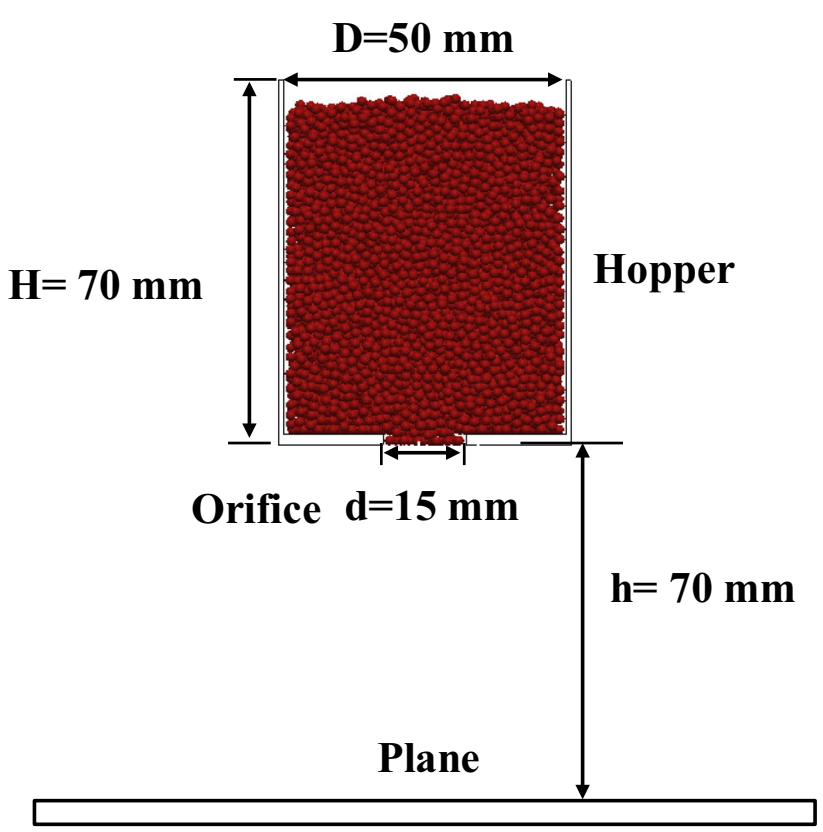

Fig. 3 Schematic for sandpile test, Orifico v.0 
Table 1 DEM input parameters

\begin{tabular}{lll}
\hline Material parameters & Symbols & Values \\
\hline Particle density $\left(\mathrm{kg} \mathrm{m}^{-3}\right)$ & $\rho$ & 2500 \\
Particle radius (m) & $R$ & 0.001 \\
Young's modulus particle (GPa) & $E_{p}$ & $0.02-200^{\mathrm{a}}$ \\
Young's modulus wall (GPa) & $E_{w}$ & 20 \\
Poisson's ratio particle & $v_{p}$ & 0.45 \\
Poisson's ratio wall & $v_{w}$ & 0.45 \\
Coefficient of restitution particle-particle & $\varepsilon_{p}$ & $0.05-0.85^{\mathrm{a}}$ \\
Static friction coefficient particle-particle & $\mu_{s, p p}$ & $0.05-0.50^{\mathrm{a}}$ \\
Rolling friction coefficient particle-particle & $\mu_{r, p p}$ & $0.05-0.45^{\mathrm{a}}$ \\
Coefficient of restitution particle-wall & $\varepsilon_{w}$ & 0.45 \\
Static friction coefficient particle-wall & $\mu_{s, p w}$ & 0.65 \\
Rolling friction coefficient particle-wall & $\mu_{r, p w}$ & 0.1 \\
\hline
\end{tabular}

${ }^{a}$ Range of values

Simulations were terminated when no more particles fell from the hopper.

Although in the real world there are no particles which have exactly identical material properties, in this work it is assumed that all the particles have the same material and contact properties. In the literature this is a common and probably a debatable practice which strongly simplifies the assignment of the DEM input parameters and therefore the number of conditions to be accounted during the calculations. The main purpose of this study is to establish a methodology and the multi-level parameters approach. Hence only four inter-particle material properties were investigated: Young's modulus $(E)$, coefficient of restitution $(\varepsilon)$, static friction coefficient $\left(\mu_{\mathrm{s}}\right)$ and rolling friction coefficient $\left(\mu_{\mathrm{r}}\right)$ as reported in Table 1 . It is recognised that other material parameters may also have an effect on the bulk particle behaviour during discharging, however to reduce the number of simulations the other material properties for particles and walls were kept constant.

\subsection{Bulk material behaviour during discharge}

Macroscopic bulk behaviours during hopper discharge have been well investigated by other researchers by looking at pressure distribution [50], FR [49,51] and contact force chains $[49,52,53]$. In this study, FR, repose angles and particle velocity at the orifice exit were considered as possible features that could be easily measured experimentally for the model validation.

\subsubsection{Flow rate $(F R)$}

FR is an important characteristic of the material flow out of a hopper. Figure 4 shows total number (continuous line) of the discharged particles as a function of the discharging time, indicating a constant FR between 0 and $3.0 \mathrm{~s}$. The FR

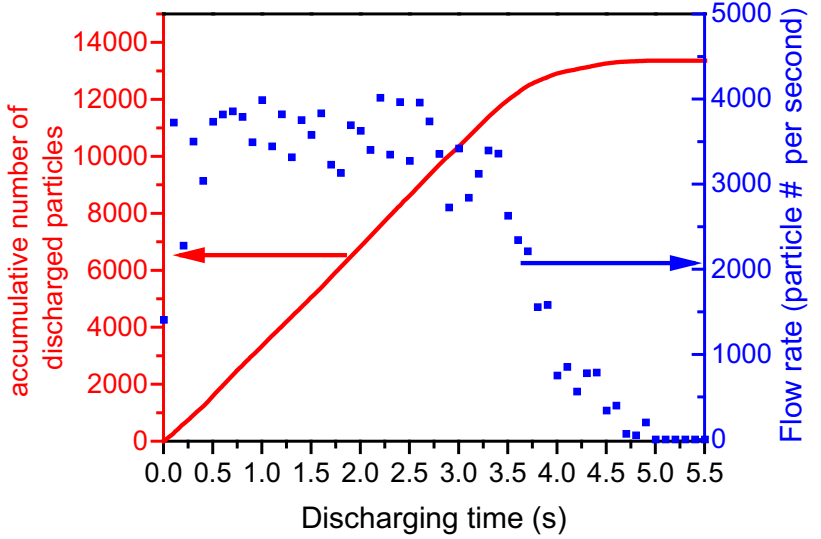

Fig. 4 Total number of particles and flow rate as functions of discharging time. Conditions for this test are: $E=0.02 \mathrm{GPa}, \varepsilon_{\mathrm{p}}=$ $0.45, \mu_{\mathrm{s}, \mathrm{pp}}=0.25, \mu_{\mathrm{r}, \mathrm{pp}}=0.25$

(square points) is defined as the number of discharged particles divided by corresponding discharging time.

\subsubsection{Repose angle}

The angle of repose reflects the flow properties of the granular material and it is associated with inter-particle friction properties. Figure 5 shows an example for the upper repose angle $\alpha$, inside the container, and the bottom repose angle $\theta$ for the discharged particles. These are calculated considering the ortho-slices along $x z$ and $y z$ planes: $\alpha=1 / 4\left(\alpha_{1}+\alpha_{2}+\right.$ $\left.\alpha_{3}+\alpha_{4}\right)$ and $\theta=1 / 4\left(\theta_{1}+\theta_{2}+\theta_{3}+\theta_{4}\right)$.

\subsubsection{Particle velocity}

Once the orifice is opened, particles start to descend driven by gravity with a flow pattern as shown in Fig. 6a. Typically using the simulation conditions, there are four discernable 
Fig. 5 Definition of the repose angles (e.g.,

$\left.\alpha=31.1^{\circ}, \theta=28.9^{\circ}\right)$.

Conditions for this test are: $E=$ $0.02 \mathrm{GPa}, \boldsymbol{\varepsilon}_{p}=0.45, \mu_{s, p p}=$ $0.25, \mu_{r, p p}=0.25$
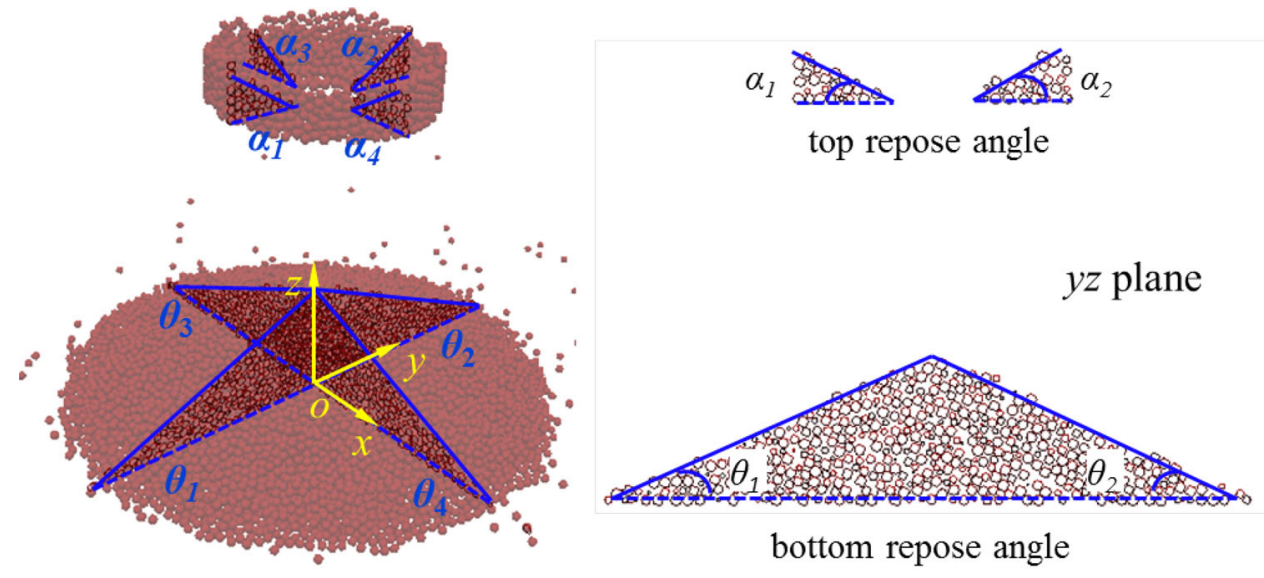

(a) average particle velocity. Only particles that are in the selected window $(\mathrm{z}=20-30 \mathrm{~mm})$ are used in the calculation. Conditions for this test are: $E=$ $0.02 \mathrm{GPa}, \varepsilon_{\boldsymbol{p}}=0.45, \mu_{\mathrm{s}, \mathrm{pp}}=$ $0.25, \mu_{\mathrm{r}, \mathrm{pp}}=0.25$

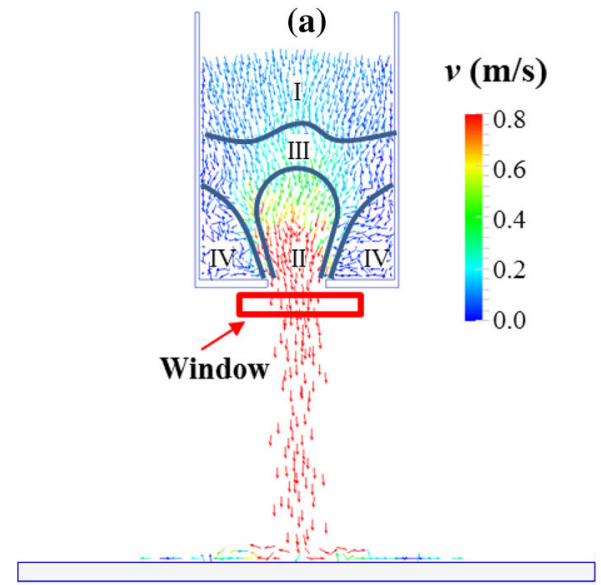

(b)

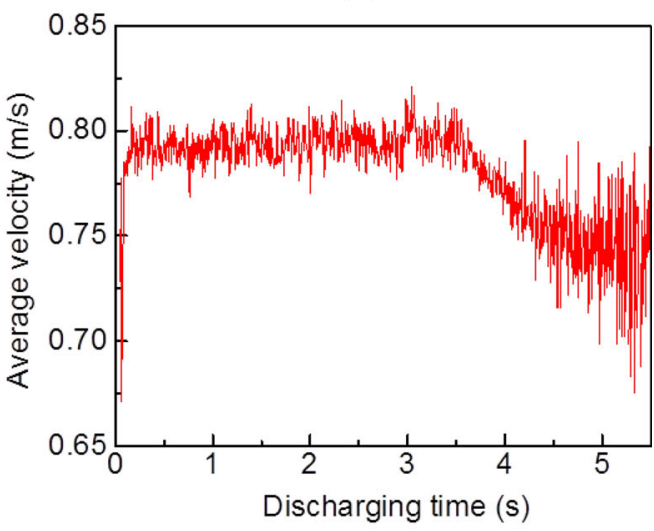

flow zones: (I) plug flow zone: particles sink relatively uniformly at the top part of the hopper, (II) converging flow zone: particles move toward orifice at high velocities, (III) transition flow zone: between plug flow zone and converging flow zone, and (IV) static zone: particles stay motionless at the corners of the hopper. To quantify the velocity for the discharging particles, the average velocity magnitude near the orifice was calculated, using a window selection zone (Fig. 6a). The average velocity near the orifice as a function of time is shown in Fig. 6 b.

Figure 7 illustrates the snapshots obtained from a simulation at different times during discharge, showing the velocity magnitude and the arrow shows the velocity vector. As the discharge proceeds, the plug flow zone is being consumed while the size of converging flow zone remains more or less the same during the discharging period, $t=0-3.0 \mathrm{~s}$. This period corresponds to a constant average velocity near the orifice (Fig. 6b). At about $t=3.5 \mathrm{~s}$, the flow starts to converge from the sides, corresponding to a decreasing average velocity near the orifice, until there are no more particles flowing out and the resulting particles form the upper repose angle.

\section{Results: sensitivity parametric study}

A parametric study was performed to obtain general trends of the system under varying input parameters (refer to Sect. 3.1 and Table 1), followed by the multi-level sensitivity analysis by using the input parameters and output quantities in the parametric study,

\subsection{Parametric studies}

\subsubsection{Effect of elastic Young's modulus E}

To examine the effect of Young's modulus $E$ on the flow behaviour for the discharging particles, we varied Young's modulus value in the range of $0.02-200 \mathrm{GPa}$. With other input parameters being held constant, three distinct simulations were carried out at three levels $(E=0.02,2.0$, and 200.0 $\mathrm{GPa})$. Figure 8 shows that $E$ in range of $0.02-200 \mathrm{GPa}$ has little influence on the final shape of the pile of material (Fig. 8a), the average velocity of particle flows near the orifice at 20-30 mm control window (Fig. 8b) and on the FR (Fig. 8c). On the other hand, the computation time varies enormously with the 


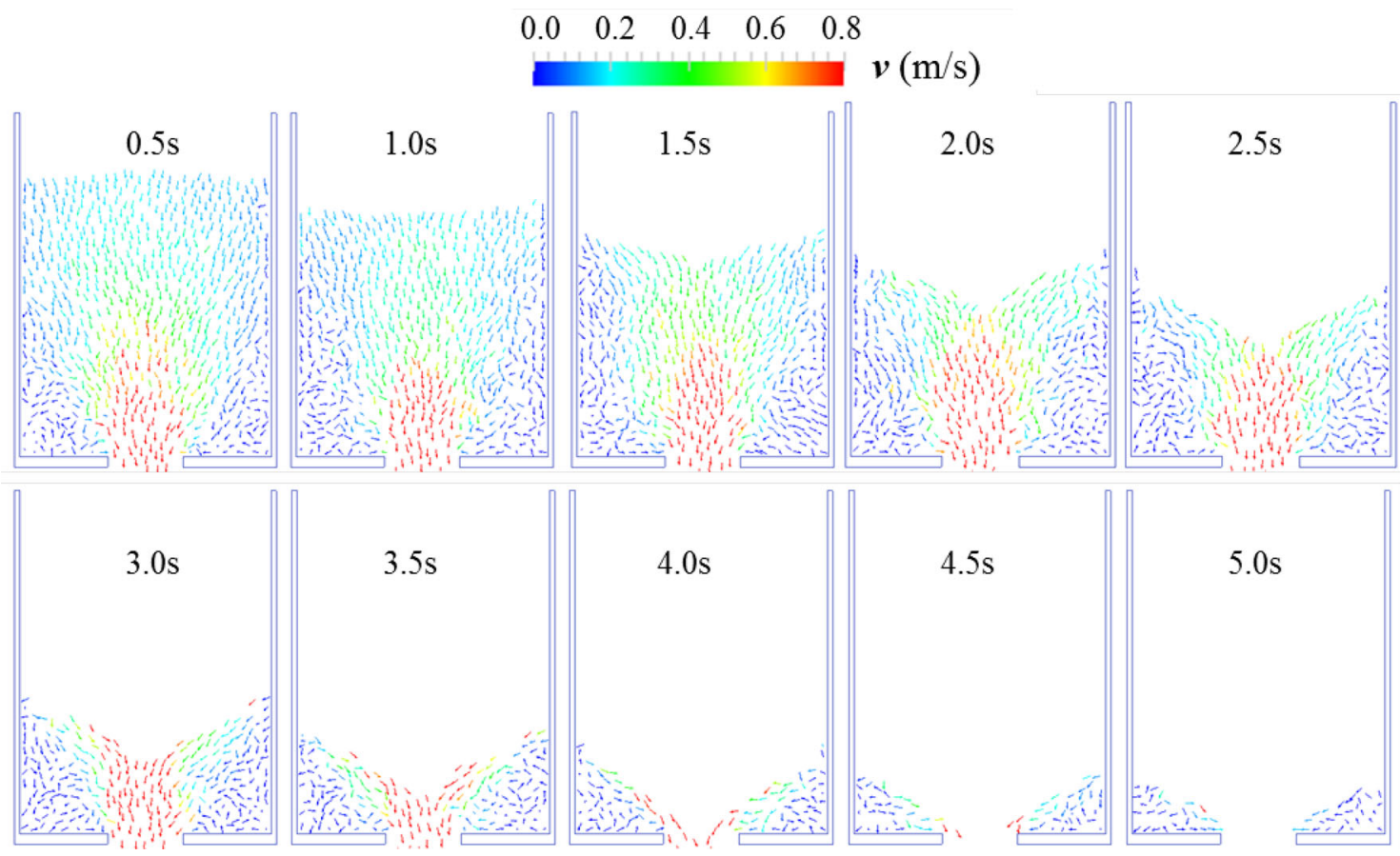

Fig. 7 Typical flow patterns within the container. Conditions for this test are: $E=0.02 \mathrm{GPa}, \varepsilon_{p}=0.45, \mu_{\mathrm{s}, \mathrm{pp}}=0.50, \mu_{\mathrm{r}, \mathrm{pp}}=0.05$

Fig. 8 Effect of Young's modulus on: a profile for the discharged material, $\mathbf{b}$ average particle velocity, and $\mathbf{c}$ number of particles discharged over the discharging time. Conditions: $\boldsymbol{\varepsilon}_{p}=0.45, \mu_{s, p p}=$ $0.50, \mu_{r, p p}=0.05$
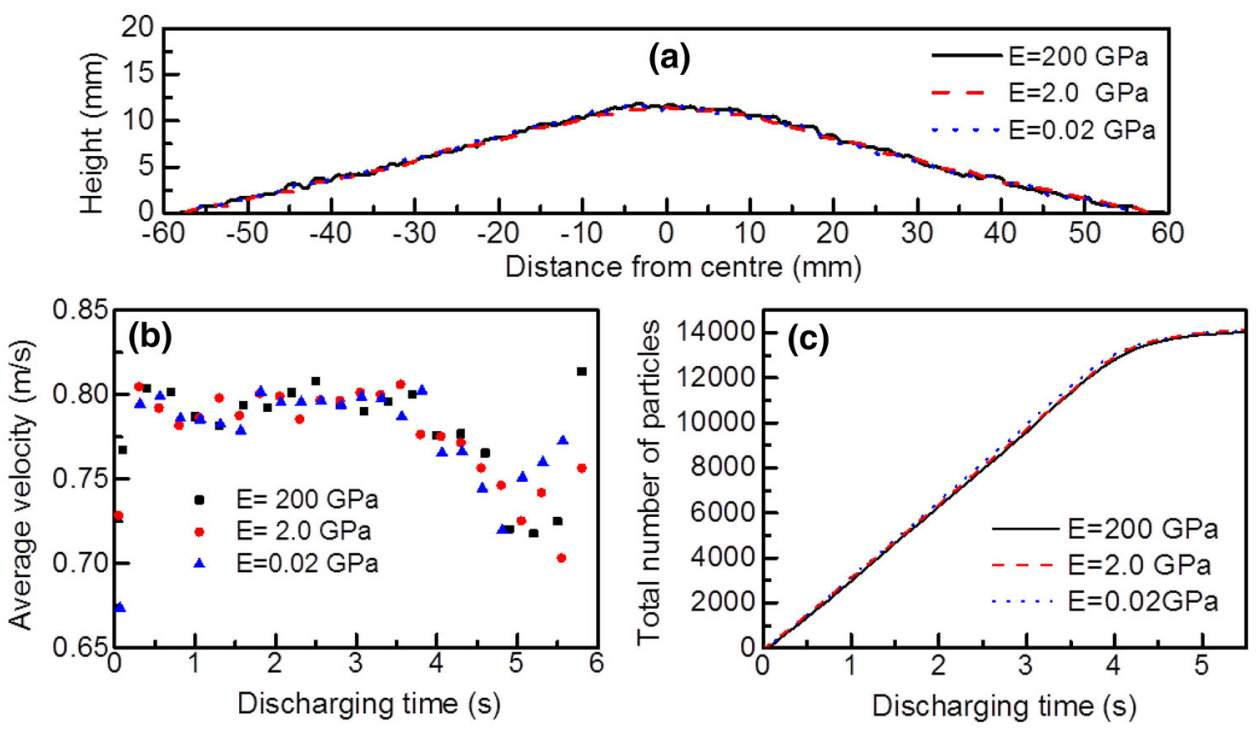

Young's modulus considering the Rayleigh's time criteria in Eq. (11). For example using 32 cores, the computation time is: $\sim 0.5 \mathrm{~h}$ for $E=0.02 \mathrm{GPa}$ (timestep $\Delta t=5 \times 10^{-6} \mathrm{~s}$ ), $\sim 3 \mathrm{~h}$ for $E=2.0 \mathrm{GPa}$ (timestep $\Delta t=5 \times 10^{-7} \mathrm{~s}$ ), and $\sim 240 \mathrm{~h}$ for $E=200 \mathrm{GPa}$ (timestep $\Delta t=5 \times 10^{-8} \mathrm{~s}$ ). This implies that a reduction in Young's modulus $E$ efficiently speeded up the simulations without altering the bulk flow behaviour. As commonly reported in the literature, to keep simulation times short the Young's modulus has often been artificially reduced as this would dramatically reduce the simulation time required. However, the choice for this parameter is important, and it has been shown that its effect depends on the actual system that is modelled. Lommen et al. [54] showed that $E$ in the range of $10^{7}-10^{11} \mathrm{~Pa}$ has almost no effect on the repose angle measurement. In other tests such as compression and penetration, a distinction can be made. They conclude that when applying a particle Young's modulus reduction in models related to the bulk stiffness and bulk restitution, shearing behaviour, and the interaction between materials and boundaries, users should be cautious and verify their approach. A more logical way is supposed to verify the effect of Young's modulus reduction for other input para- 
Fig. 9 Effect of restitution coefficient $\varepsilon_{p}$ on: a profile for the discharged material, $\mathbf{b}$ repose angle (bottom angle $\theta$ ) versus the coefficient of restitution, and $\mathbf{c}$ flow rate versus the coefficient of restitution. Simulations in $\mathbf{b}$ and $\mathbf{c}$ were conducted at $\mu_{\mathrm{r}, \mathrm{pp}}=0.45$
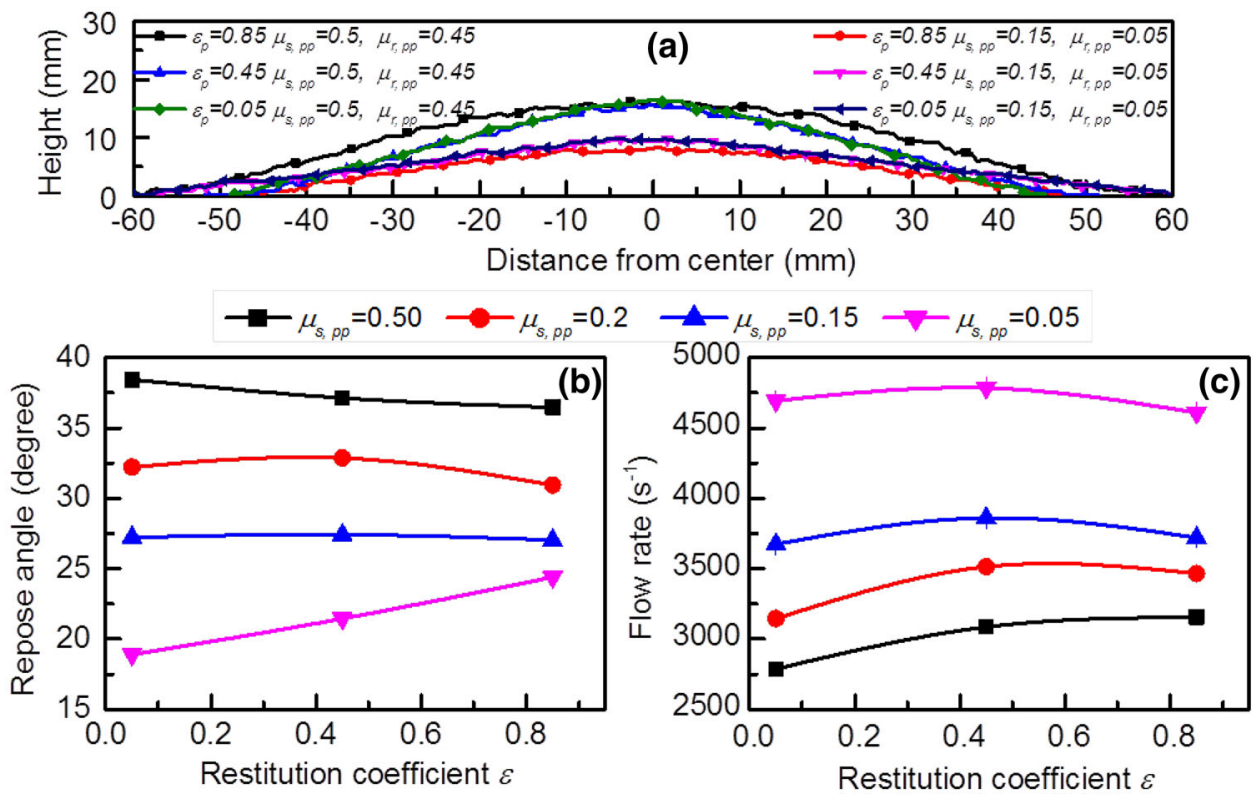

meters combinations at least covering the corner and centre points in the design of simulations (DoSs) space. However, this was not addressed in the current scope due to computation source limitation.

As the model outputs shown in Fig. 8 were shown to be insensitive with respect to $E$, the smallest value of this parameter could be chosen purely on the basis of reducing computational time. A simulation matrix was constructed for all subsequent simulations and the parameter levels chosen within this matrix were: $\mu_{s, p p}=0.05,0.15,0.25,0.50, \mu_{r, p p}=$ $0.05,0.25,0.45$, and $\varepsilon_{p}=0.05,0.45,0.85$. A full factorial design approach was used comprising $4 \times 3^{2}=36$ distinct simulations in total. Coverage of $\mu_{s, p p}$ was extended to four levels due to the significance of this parameter in our pretest simulation outcomes. This effect will be subsequently discussed.

\subsubsection{Effect of coefficient of restitution}

Figure 9a shows the profiles for the discharged pile simulated with different coefficients of restitution for both low $\left(\mu_{s, p p}=\right.$ $\left.0.15 \mu_{r, p p}=0.05\right)$ and high $\left(\mu_{\mathrm{s}, \mathrm{pp}}=0.5 \mu_{\mathrm{r}, \mathrm{pp}}=0.45\right)$ friction coefficients. Varying restitution coefficient in the range of $\varepsilon_{p}=0-0.45$ does not lead to any obvious difference in the shape of the discharged pile. However, when $\varepsilon_{p}$ increases to 0.85 the profile does slightly change. Figure $9 \mathrm{~b}, \mathrm{c}$ show that the restitution coefficient has little influence on the bottom repose angle or on the FR. For the repose angle, at a lower friction level, the repose angle increases as restitution coefficient increases, at higher friction levels the repose angle decreases as the coefficient of restitution coefficient increases. For FR, which can be considered as an inverse variable against repose angle, the influence behaves in an opposite manner. There is almost no effect of the coefficient of restitution on the flowability for a medium $\varepsilon_{p}$ value. It is apparent that the effects of friction and coefficient of restitution are cross correlated. An investigation of the cross correlation of the parameters in the system is thus imperative and this will be presented in a following section.

\subsubsection{Effect of static and rolling friction coefficients}

Figure 10 shows the effect of the static and rolling friction coefficients on the profile for the discharged pile, on the repose angle and on the FR. From Fig. 10a, it can be seen that with increasing of static friction or rolling friction the height of the sand pile increases and the width of sandpile decreases. It also could be seen that the effects of static and rolling friction have a combination effect. When the static friction $\mu_{s, p p}$ is low $\left(\mu_{s, p p}=0.05\right)$, the effect of rolling friction is not significant; when the static friction $\mu_{s, p p}$ is high $\left(\mu_{s, p p}=0.5\right)$, the effect of rolling friction is more significant. Even when $\mu_{r, p p}$ is very low $\left(\mu_{r, p p}=0.05\right)$, the effect of static friction is significant. This implies that the effect of rolling friction is secondary to that of static friction. In effect, the repose angle increases with increasing of static friction (Fig. 10b) or rolling friction (Fig. 10d). The FR decreases as the static friction coefficient and to a lesser extent, the rolling friction coefficient, are increased. Simply, and evidently, the flowability of granular materials decreases as inter-particle friction coefficients increase. The effect of static friction appears to be stronger than that of the rolling friction, upon the comparison of the slopes of the curves; indicating a stronger effect of the flowability of the granular materials. Moon et al. [55] reported that friction must 
Fig. 10 Effect of static and rolling friction coefficients: a profile for the discharged material, all the simulations run with constant $\varepsilon_{\mathrm{p}}=0.45$, b repose angle $\theta$ versus static friction coefficient, $\mathbf{c}$ flow rate versus static friction coefficient, d repose angle $\theta$ versus rolling friction coefficient, and e flow rate versus rolling friction coefficient
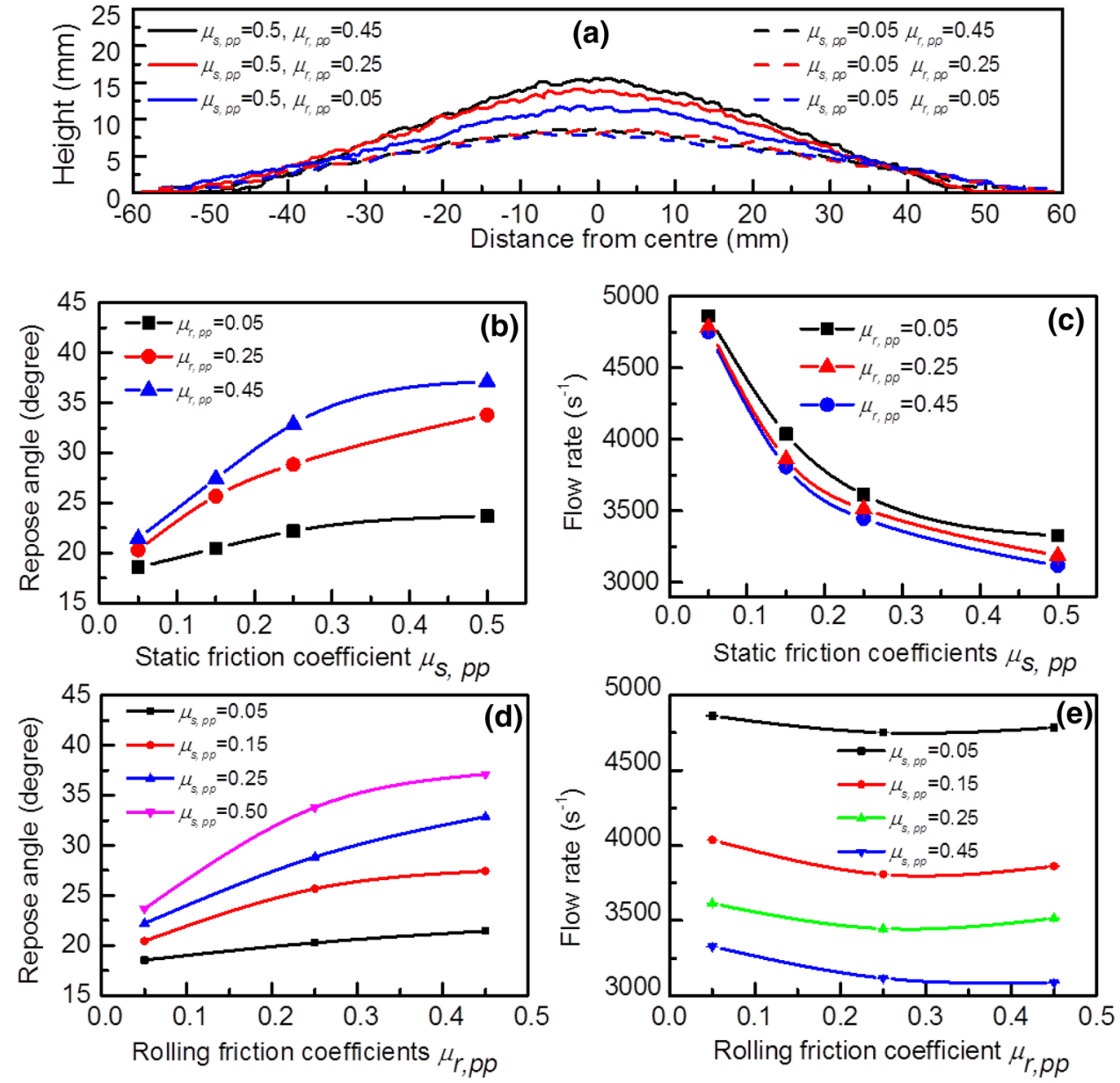

be included in every simulation because it dissipates energy, reduces grain mobility, and increases overall collision rate.

It can clearly be seen that from the simple case here presented, a parametric DEM study without using a robust statistical approach could lead to major complications when trying to compare or evaluate the effect of the input parameters on output results. This would be even more challenging if higher number of input parameters were required as more complex contact models were considered.

\subsection{Parameter sensitivity studies}

\subsubsection{Level 1 analysis}

In Sect. 4.1, 36 distinct sandpiles were simulated using the DoSs matrix. The measured top $(\alpha)$ and bottom $(\theta)$ angle of repose observed at the end of the simulation were fitted to the following power law relationship:

$\alpha$ or $\theta=A \cdot \varepsilon_{p}^{b} \cdot \mu_{s, p p}^{c} \cdot \mu_{r, p p}^{d}$,

where $A, b, c$ and $d$ are fitted empirical constants. For each parameter estimation procedure, all four measured angles for the top and bottom angles of repose were included in the fitting, rather than the average. This enabled an understanding of the impact of angle variance for each of the 36 simulations to be understood in context of the fitting. Parity plots for the results of the fitting process are shown in Fig. 11.

As part of the above parameter estimation results, a lack of fit analysis was carried out to assess the impact of measured angle differences (the so-called 'experimental error') against model error. The variance ratio (model error:experimental error) was found to be 9.02 and 122.3 for the upper and lower angles of repose, respectively. This is significantly above the critical $t$-value threshold at $95 \%$ confidence for this system of \pm 1.96 . Therefore it can be concluded that the parameters estimated in the model are influenced by differences between the input parameter set points rather than variances between the four angles at individual measurements.

Table 2 below reports the estimated parameters from the fitting process and their associated $95 \%$ confidence intervals. Larger magnitudes of power indices imply a greater dependency of model responses with respect to the corresponding material parameters.

The results in Table 2 suggest that $\mu_{r, p p}$ and $\mu_{s, p p}$ both play a significant role in determining the final repose angle 
Fig. 11 Parity plots for top and bottom angles of repose in the sand-pile test. Blue squares, red squares green squares purple squares denotes first, second, third and fourth angle measurements, respectively. (Color figure online)
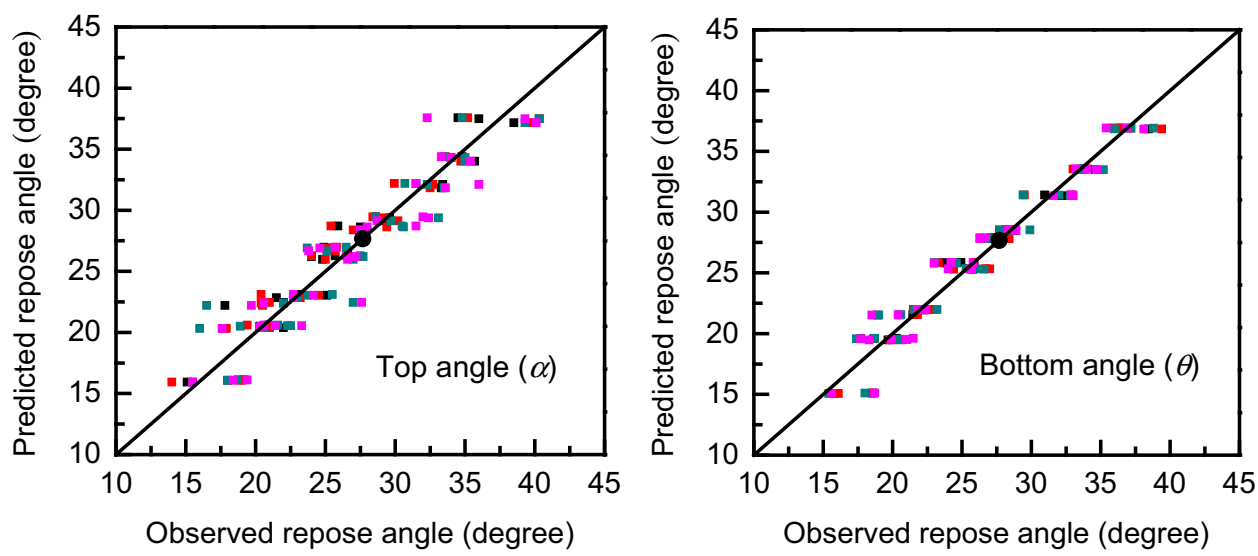

Table 2 Parameter estimation results for power law model fitting to relate DEM input parameters to angle of repose responses

\begin{tabular}{lcccc}
\hline Parameters & \multicolumn{2}{l}{ Upper angle of repose $(\alpha)$} & \multicolumn{2}{l}{ Lower angle of repose $(\theta)$} \\
\cline { 2 - 5 } & Estimates & $95 \%$ Confidence interval & Estimates & $95 \%$ Confidence interval \\
\hline $\mathbf{A}$ & 49.53 & 1.77 & 49.40 & 1.25 \\
$\mathbf{b}\left(\rightarrow \varepsilon_{\mathrm{p}}\right)$ & 0.04 & 0.01 & 0.01 & 0.01 \\
$\mathbf{c}\left(\rightarrow \mu_{\mathrm{s}}\right)$ & 0.22 & 0.02 & 0.23 & 0.01 \\
$\mathbf{d}\left(\rightarrow \mu_{\mathrm{r}}\right)$ & 0.15 & 0.01 & 0.16 & 0.01 \\
\hline
\end{tabular}

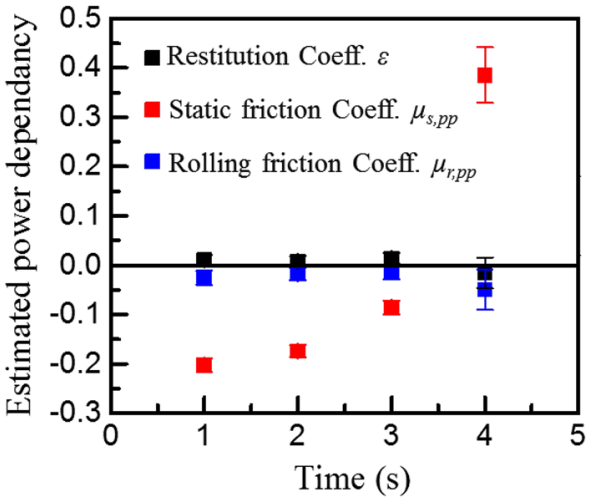

Fig. 12 Estimated power dependencies of varied DEM model parameters on particle flow rate out of the hopper as a function of discharging time. Error bars denote asymptotic $95 \%$ confidence intervals calculated from the parameter estimation process

in each simulation whilst $\varepsilon_{p}$ has a very weak influence. The values of $\mu_{s, p p}$ is considerably more significant than $\mu_{r, p p}$ in determining the lower angle. This result is consistent with the findings in [31].

In addition, the impact of model parameters in the simulation matrix can be assessed against FR at various points during the simulations. Using the hopper FR measurements (number of particles $\mathrm{s}^{-1}$ ) as exemplified in Fig. 3, the power law relationship in Eq. (19) was applied at time points, $t=1,2,3,4 \mathrm{~s}$ during the simulation. Estimated parameters from this analysis are shown in Fig. 12.
The results in Fig. 12 show a clear dependency of FR on $\mu_{s, p p}$ throughout the discharging time, whilst $\mu_{r, p p}$ and $\varepsilon_{p}$ remain insignificant in their contribution. The role of $\mu_{s, p p}$ also appears to shift with time. Initially, choice of $\mu_{s, p p}$ value has a negative power influence on FR (i.e., larger $\mu_{s, p p}$ results in a slower FR) however this shifts to a positive relationship towards the end of the discharging time. This suggests that the particle flow mechanism out of the hopper changes; this is a shift from core flow under gravity to flow from the sides of the hopper.

To investigate further, the FR profiles (e.g., Fig. 4) were analysed for a smaller range of experiments. The five simulations chosen encompassed the high-low values for $\mu_{s}$ and $\mu_{r}(0.05 \rightarrow 0.50$ and $0.05 \rightarrow 0.45$, respectively $)$ as well as a centre point $\left(\mu_{s, p p}\right.$ and $\left.\mu_{r, p p}=0.25\right)$. To model the flow profiles and discriminate further behaviours as a function of DEM inputs parameters, the following three-parameter, time-dependent empirical model was set up:

$\mathrm{FR}(t)=\frac{\mathrm{FR}_{0}}{1+(K \cdot t)^{n}}$,

where FR denotes flow rate, $\mathrm{FR}_{0}$ is the estimated initial FR at time $t=0 \mathrm{~s}, K$ is a characteristic time for the onset of FR decrease, $n$ is the rate of decline of the FR. The results for the five analysed simulations are shown in Fig. 13 below:

Examination of Fig. 13 shows the time-dependent model approach describes the simulation trends well in all cases. The results of the parameter estimates for the model application are shown in Table 3. 


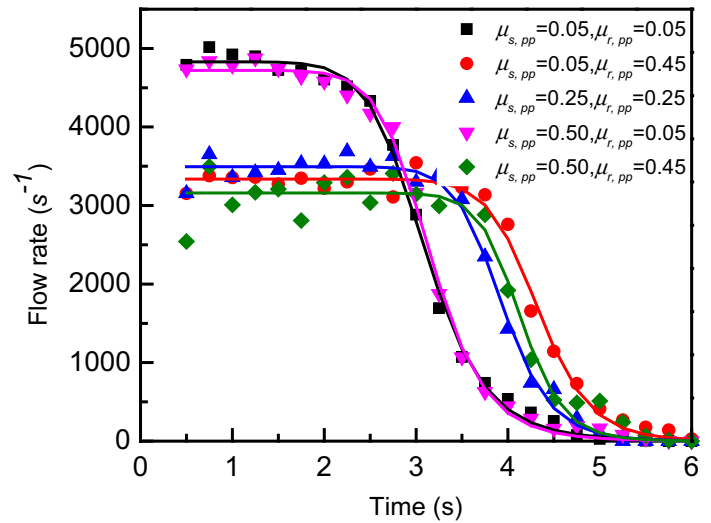

Fig. 13 Particle flow rate out of the hopper versus discharging time for five select simulations. $\varepsilon_{p}=0.45$ in all cases; solid lines denote model prediction using Eq. (20)

Table 3 Parameter estimation results for fitting of time-dependent empirical model to flow rate data

\begin{tabular}{lllll}
\hline$\mu_{s, p p}$ & $\mu_{r, p p}$ & $\mathrm{FR}_{0}\left(\mathrm{~s}^{-1}\right)$ & $K\left(\mathrm{~s}^{-1}\right)$ & $n(-)$ \\
\hline 0.05 & 0.05 & $4830 \pm 84$ & $0.322 \pm 0.004$ & $9.43 \pm 0.87$ \\
0.5 & 0.05 & $3340 \pm 77$ & $0.231 \pm 0.001$ & $15.3 \pm 2.60$ \\
0.25 & 0.25 & $3500 \pm 96$ & $0.254 \pm 0.004$ & $14.9 \pm 2.61$ \\
0.05 & 0.45 & $4720 \pm 89$ & $0.317 \pm 0.004$ & $10.6 \pm 1.15$ \\
0.5 & 0.45 & $3160 \pm 156$ & $0.242 \pm 0.006$ & $17.8 \pm 3.85$ \\
\hline
\end{tabular}

In Table 3, a significantly higher $\mathrm{FR}_{0}$ parameter is estimated in the simulations using $\mu_{s, p p}=0.05$ in comparison to $\mu_{s, p p}=0.25$ and 0.5 . This again shows the strong influence of $\mu_{s, p p}$ on the system but also suggests that the impact of $\mu_{s, p p}$ on this result is non-linear as the differences between $\mu_{s, p p}=0.05$ and 0.25 are considerably greater than $\mu_{s, p p}=0.25$ and 0.50 . Accordingly, the $K$ parameter matches the estimates for $\mathrm{FR}_{0}$; faster initial flows result in a faster emptying of the central core leading to an earlier onset of FR decline.

In addition, the rate of decline index, $n$, shows an influence of $\mu_{s, p p}$. Where $\mu_{s, p p}=0.05$, this rate is much slower than the other results, suggesting it takes longer for the side sections of the hopper to empty. This is logical as in the $\mu_{s, p p}=0.05$ systems, a greater final magnitude of particles are found to have flowed out of the hopper by the end of the simulation; an observation matched by a smaller final upper angle of repose.

Average particle velocity against discharge time at the orifice window was also investigated in the same manner as particle FR. Between different parameter combinations, the differences in particle velocity during the constant FR period were much less clear. This was owed to lower signal to noise ratio and also smaller differences in magnitude of velocity between the simulations. As a result total FR of particles was chosen as a clearer descriptor to characterise differences in simulation outputs as a function of parameter choice.

\subsubsection{Level 2 analysis}

In this analysis, an approach is taken whereby bulk observed parameters (e.g., FR, angle of repose) are analysed as a function of sensitivities derived from the equations of motion for mass [Eq. (2)] and moment of inertia [Eq. (3)]. In order to understand the mathematical structure of these sensitivities, Eqs. (2) and (3) are firstly re-examined as functions of $\varepsilon_{p}, \mu_{r, p p}$ and $\mu_{s, p p}$ as follows:

$\mathrm{m}_{\mathrm{i}} \frac{\mathrm{d} \boldsymbol{v}_{\mathrm{i}}}{\mathrm{dt}}=\sum\left(\boldsymbol{F}_{\mathrm{ij}}^{\mathrm{n}}+\boldsymbol{F}_{\mathrm{ij}}^{\mathrm{t}}\right)+\mathrm{m}_{\mathrm{i}} \boldsymbol{g}=f\left(\varepsilon, \mu_{s}\right)$,

$I_{\mathrm{i}} \frac{\mathrm{d} \boldsymbol{\omega}_{\mathrm{i}}}{\mathrm{dt}}=\sum\left(\boldsymbol{R}_{\mathrm{i}} \times \boldsymbol{F}_{\mathrm{ij}}^{\mathrm{t}}-\boldsymbol{\tau}_{\mathrm{ij}}^{\mathrm{r}}\right)=f\left(\varepsilon, \mu_{s}, \mu_{r}\right)$.

In Eq. (22), $\mu_{s}$ acts as a function due to the influence on the value of $\boldsymbol{F}_{i j}^{t}$ as a result of the relationship in Eqs. (6), (21) and (22) can be expanded using the expressions for $\boldsymbol{F}_{i j}^{n}$ as $\boldsymbol{F}_{i j}^{t}$ as defined in Eqs. (4) and (5). Response sensitivities of Eqs. (21) and (22) can be calculated analytically by differentiating these expressions with respect to the parameter of interest $\left(\varepsilon_{p}, \mu_{r, p p}\right.$ and $\left.\mu_{s, p p}\right)$ :

$$
\begin{aligned}
& \frac{\mathrm{d}\left(\mathrm{m}_{\mathrm{i}} \frac{\mathrm{d} \boldsymbol{v}_{\mathrm{i}}}{\mathrm{dt}}\right)}{\mathrm{dP}}=\frac{\mathrm{d}\left(\sum\left(\boldsymbol{F}_{\mathrm{ij}}^{\mathrm{n}}+\boldsymbol{F}_{\mathrm{ij}}^{\mathrm{t}}\right)+\mathrm{m}_{\mathrm{i}} \boldsymbol{g}\right)}{\mathrm{dP}}, \\
& \frac{\mathrm{d}\left(I_{\mathrm{i}} \frac{\mathrm{d} \omega_{\mathrm{i}}}{\mathrm{dt}}\right)}{\mathrm{dP}}=\frac{\mathrm{d}\left(\sum\left(\boldsymbol{R}_{\mathrm{i}} \times \boldsymbol{F}_{\mathrm{ij}}^{\mathrm{t}}-\boldsymbol{\tau}_{\mathrm{ij}}^{\mathrm{r}}\right)=f\left(\varepsilon, \mu_{s}, \mu_{r}\right)\right)}{\mathrm{dP}} .
\end{aligned}
$$

The analytical descriptions of the sensitivities in Eqs. (23) and (24) are extensive, and also contain velocity $\left(v_{i j}^{n}\right.$ and $\left.v_{i j}^{t}\right)$ and overlap $\left(\delta_{i j}^{n}\right.$ and $\left.\delta_{i j}^{t}\right)$ inputs, which can vary significantly on an individual particle basis over a given simulation. Hence for the Level 2 analysis, which aims to link bulk observations to fundamental model structure, a pragmatic approach is taken utilising simplified, general form descriptions of the response sensitivities with respect to the input parameters. The mathematical appearance of these derivatives is shown in Table 4.

In Table 4, it is seen that $\mu_{r}$ and $\mu_{s}$ take the form of simple linear functions whilst the function for $\varepsilon_{p}$ is distinctly non-linear and also has a more complex derivative function. These derivatives are utilised in the generation of the two parameter sensitivity matrices for the PCA; one for the equation of motion for mass and moment of inertia, respectively. Each matrix therefore comprises 4 columns (3 parameters and 1 bulk response) and 36 rows to account for all parameter combinations in the DoSs. Equations (25) and (26) below illustrates the appearance of the sensitivity matrix, $S_{i}$, 
Table 4 Generalised appearance and derivatives of input parameter functions in the fundamental DEM equations

\begin{tabular}{lllll}
\hline Parameters & $\begin{array}{l}\text { General form in both } \\
\text { equations }\end{array}$ & $\begin{array}{l}\text { Derivative of general } \\
\text { form }\left(\delta f / \delta P_{k}\right)\end{array}$ & $\begin{array}{l}\text { General derivative in } \\
\text { equation of motion } \\
\text { for mass [Eq. (21)] }\end{array}$ & $\begin{array}{l}\text { General deriva- } \\
\text { tive in equation of } \\
\text { motion for moment } \\
\text { of inertia [Eq. (22)] }\end{array}$ \\
\hline$\varepsilon_{p}$ & $\frac{\ln \varepsilon_{p}}{\left(\left(\ln \varepsilon_{p}\right)^{2}+\pi^{2}\right)^{0.5}}$ & $\frac{\ln \varepsilon_{p}}{\left(\left(\ln \varepsilon_{p}\right)^{2}+\pi^{2}\right)^{0.5}}$ & $\frac{\mu_{s} \cdot \pi^{2}}{\left(\varepsilon_{p} \cdot\left(\left(\ln \varepsilon_{p}\right)^{2}+\pi^{2}\right)\right)^{1.5}}$ & $\frac{\mu_{s} \cdot \mu_{r} \cdot \pi^{2}}{\left(\varepsilon_{p} \cdot\left(\left(\ln \varepsilon_{p}\right)^{2}+\pi^{2}\right)\right)^{1.5}}$ \\
$\mu_{s}$ & $\mu_{\mathrm{s}}$ & 1 & $\frac{\ln \varepsilon_{p}}{\left(\left(\ln \varepsilon_{p}\right)^{2}+\pi^{2}\right)^{0.5}}$ & $\frac{\mu_{r} \cdot \ln \varepsilon_{p}}{\left(\left(\ln \varepsilon_{p}\right)^{2}+\pi^{2}\right)^{0.5}}$ \\
$\mu_{r}$ & $\mu_{\mathrm{r}}$ & 1 & 0 & $\frac{\mu_{s} \cdot \ln \varepsilon_{p}}{\left(\left(\ln \varepsilon_{p}\right)^{2}+\pi^{2}\right)^{0.5}}$ \\
\hline
\end{tabular}

\begin{tabular}{|c|c|c|c|c|}
\hline \multirow[t]{2}{*}{ Equation of motion } & \multirow{2}{*}{$\begin{array}{l}\text { Eigenvalues } \\
\text { Principal components }\end{array}$} & \multicolumn{3}{|c|}{ Eigenvectors } \\
\hline & & $\varepsilon_{p}$ & $\mu_{s}$ & $\mu_{r}$ \\
\hline \multirow[t]{2}{*}{ Mass } & $\Lambda_{1}=9.63$ & 0.05 & -0.90 & 0 \\
\hline & $\Lambda_{2}=0.72$ & -0.99 & -0.08 & 0 \\
\hline \multirow[t]{2}{*}{ Moment of inertia } & $\Lambda_{1}=2.36$ & -0.04 & -0.46 & 0.88 \\
\hline & $\Lambda_{2}=1.34$ & 0.02 & -0.88 & -0.46 \\
\hline
\end{tabular}

Table 5 Impact of DEM parameters in terms of equation of motion for mass and moment of inertia derivatives on final bottom angle of repose $(\theta)$ in simulations $\mu_{s, p p}$ and the FR response is consistent with the empirical model findings in Fig. 12.

\subsubsection{Level 3 analysis}

As with the Level 2 analysis, the Level 3 approach utilises the derivatives of the fundamental DEM equations in Eqs. (2) and (3). The key difference is that the full analytical derivative descriptions derived from Eqs. (23) and (24) are used in this analysis rather than the generalised form used in the Level 2 analysis (see Table 4). As a result, input variables such as overlap distance $\left(\delta_{i j}^{n}\right.$ and $\left.\delta_{i j}^{t}\right)$, velocity $\left(v_{i j}^{n}\right.$ and $\left.v_{i j}^{t}\right)$ and angular velocity $\left(\omega_{i j}\right)$ are needed as inputs for the sensitivity calculations using these derivatives.

To achieve the above, the Level 3 analysis does not rely on using the bulk model responses that were used in the Level 2 analysis. Instead, the analysis relies on selecting a range of feasible velocities and overlaps that could feature within the system under study. An estimation of the range of values found for these variables in the sand-pile test was ascertained by analysis of a selection of outputs files taken from the simulation matrix used in this study. The input variable levels chosen for calculation were found to be thus:

- $v_{i j}^{n}=0.0001,0.01$ and $1 \mathrm{~m} \mathrm{~s}^{-1}$,

- $v_{i j}^{t}=0.0001,0.01$ and $1 \mathrm{~m} \mathrm{~s}^{-1}$,

- $\omega_{i j}=0.01,1$ and $100 \mathrm{rad} \mathrm{s}^{-1}$,

- $\delta_{i j}^{n}$ and $\delta_{i j}^{t}=0.1,1$ and $10 \%$ of particle diameter.

In the above, $\delta_{i j}^{n}$ and $\delta_{i j}^{t}$ are set equal in all cases. This approach is referred to as an a priori approach as these values can, in theory, be input into this analysis prior to running any 
Fig. 14 Normalised norms of the parameter sensitivity matrix for $\mu_{r, p p}, \varepsilon_{p}, E$ and $\mu_{s, p p}$ using different combinations of $\mu_{r, p p}$ and $\mu_{s, p p}$ values: a equation of motion for mass response, and $\mathbf{b}$ equation of motion for moment of inertia response
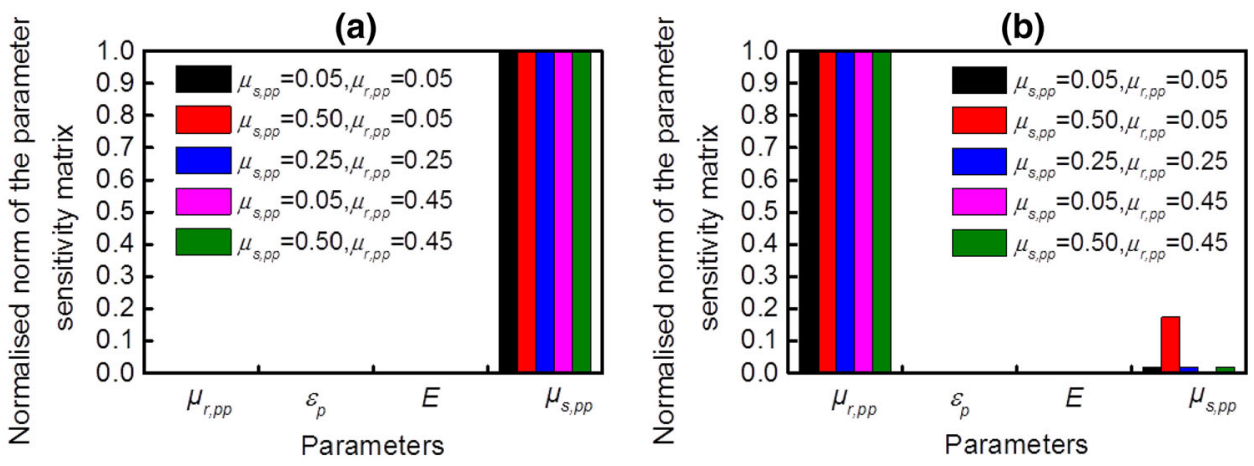

DEM simulations, to gauge model response sensitivities. The analysis in this section was carried out in Microsoft Excel.

The input parameter combinations for the five simulations analysed in Fig. 12 were used in this analysis. This was done for simplicity, as these simulations exemplify the important parameters in the system. For each of the five simulations, the above input variables were applied to the model response sensitivities in all possible combinations $\left(3^{4}=81\right.$ in total). Derivatives for equations of mass and moment of inertia were calculated with respect to $\varepsilon_{p}, \mu_{s, p p}, \mu_{r, p p}$ and $E$. Young's modulus was included despite the fact that it was previously shown to be insignificant over the range tested. This was done to provide an additional cross check for effectiveness of the Level 3 analysis method.

For a given combination of parameters, this yielded a parameter sensitivity matrix of $81 \times 4$ for each of the two model responses. These matrices were then analysed using the norm of the parameter sensitivity matrix shown in Eq. (17), as opposed to the PCA approach. The authors note that PCA could be used here, but the norm of the parameter sensitivity matrix is demonstrated instead to show the applicability of conveniently lumping sensitivities to deliver the same level of understanding as was shown in the Level 2 analysis.

To demonstrate the importance of each parameter, the norms of the parameter sensitivity matrix were themselves normalised for both of the model responses. Figure 14 shows the result of this process for all five simulation conditions.

In Fig. 14, $\mu_{s, p p}$ is clearly seen to be the dominant parameter when considering the equation of motion for mass, regardless of the five input parameter conditions. For inertia model response, the dominant parameter is $\mu_{r, p p}$, although a smaller but significant contribution is seen from $\mu_{s, p p}$. Both outcomes are consistent with the semi-empirical findings using the PCA method. Prior to normalisation, it is noted that the sensitivities for $\mu_{s, p p}$ in the equation of motion for mass are, on average, two-three orders of magnitude greater than those of $\mu_{r, p p}$ in the equation of motion for moment of inertia. This would suggest that a shift in parameter values has a much greater impact on contact force calculations in this system, rather than torque, thus showing the dominance of $\mu_{s, p p}$ in the sandpile test under study.

To further understand parameter significance in the system, the levels of cross-correlation between the parameters can also be assessed using Eq. (17) and are reported in Table 6.

In addition to the small contribution that $E$ and $\varepsilon_{\boldsymbol{p}}$ make to the sensitivity of the responses within the DEM simulation, the two parameters are also completely cross correlated. The other parameter relationships for both model responses show no significant cross correlation over the test conditions used to calculate the sensitivities. This is important for the $\mu_{s, p p}$ and $\mu_{r, p p}$ relationship in the equation of motion for moment of inertia; $\mu_{s, p p}$ is shown to play a minor role in this instance and here is confirmed to be largely free of influence from $\mu_{r, p p}$. The cross correlation matrix was very similar when the other parameter combinations investigated in Fig. 14 were used as inputs.

It is observed that the three levels of analysis used all point to very similar conclusions, namely that $\mu_{s, p p}$ has the largest impact on the DEM simulation, whether it be via bulk observation analysis or the use of a priori inputs to underpinning models applied in DEM. Consistent observations between the methods are also true for the roles of $\mu_{r, p p}$ and $\varepsilon_{p}$.

In terms of computational time, the Level 3 approach is the fastest to implement. Once the user has set up a calculation method for the various derivatives and sensitivities for the system, parameter significance can be quickly ascertained over a range of overlaps and velocities. Parameters themselves however have to be changed between each assessment. The drawback to the Level 3 lies in the selection of appropriate input velocities and overlaps. This ideally should be scoped from DEM simulation results, as was carried out in this paper. Levels 1 and 2 approaches are more time consuming as they require the completion of lengthy DEM simulations to obtain the bulk outputs, but the sensitivity analysis process is much faster and can factor all combinations of input parameters explored simultaneously. In essence, a combinatorial approach between these methods 
Table 6 Example

cross-correlation matrix for

$\mu_{r, p p}, \varepsilon_{p}, E$ and $\mu_{s, p p}$

parameters for test conditions

when $\mu_{r, p p}=0.05, \varepsilon_{p}=$

$0.45, E=2 \times 10^{7} \mathrm{~Pa}$ and

$\mu_{s, p p}=0.5$

\begin{tabular}{|c|c|c|c|c|c|c|c|c|c|}
\hline \multicolumn{5}{|c|}{ Equation of motion for mass response } & \multicolumn{5}{|c|}{ Equation of motion for moment of inertia response } \\
\hline & $\mu_{r, p p}$ & $\varepsilon_{p}$ & $E$ & $\mu_{s, p p}$ & & $\mu_{r, p p}$ & $\varepsilon_{p}$ & $E$ & $\mu_{s, p p}$ \\
\hline$\mu_{r, p p}$ & 0 & 0 & 0 & 0 & $\mu_{r, p p}$ & 1 & 0 & 0 & 0 \\
\hline$\varepsilon_{p}$ & 0 & 1 & 0 & 0 & $\varepsilon_{p}$ & -0.344 & 1 & 0 & 0 \\
\hline E & 0 & -1 & 1 & 0 & $E$ & 0.344 & -1 & 1 & 0 \\
\hline$\mu_{s, p p}$ & 0 & -0.147 & 0.147 & 1 & $\mu_{s, p p}$ & 0.499 & -0.090 & 0.090 & 1 \\
\hline
\end{tabular}

could be fruitful. A small matrix of initial DEM simulations could be used to scope system behaviours and feed into the Level 3 analysis. Understanding from this can then inform a select number of targeted DEM simulations where input parameters are varied. These can then be analysed using the Levels 1 and 2 approaches. Concerning the matrix operations for all three levels, these usually require just a few seconds using aforementioned statistics analysis commercial packages.

\section{Conclusions}

In this study, we proposed the application of a statistically driven methodology to understand the impact of DEM input parameters, such as Young's modulus $(E)$, restitution coefficient $\left(\varepsilon_{p}\right)$, static friction coefficient $\left(\mu_{\mathrm{s}, \mathrm{pp}}\right)$ and rolling friction coefficient $\left(\mu_{\mathrm{r}, \mathrm{pp}}\right)$, on the bulk response for a simple system. The repose angle and FR and FR bulk responses were found to show significant differences as a function of choice of input parameters, whilst average particle velocity at the hopper orifice was found to be much less sensitive. The link between input parameter and output responses has been considered at three levels of complexity, using a statistical approach. The statistical analysis results showed consistency with the DEM direct observations. For example, it was shown that static friction coefficient $\mu_{s}$ plays a primary role on both angle of reposes and FR, followed by a less but still significant role for the rolling friction coefficient $\mu_{r}$. The restitution coefficient $\varepsilon_{p}$ was found to have an insignificant impact for this system. These observations are likely to be dependent on the system of study and their relative importance may be different on a case by case basis. The proposed statistical analysis method could be very helpful to evaluate more complicated systems including a higher number of input parameter.

A practical routine has been presented to investigate the link between bulk behaviour and single particle properties at three levels:

- Level 1 The simplest models or functions are built to describe the link between input parameters and output bulk responses. The empirical relations contain quali- tative and quantitative relations between the input and output quantities. Practically, after carrying out the parametric DEM simulations under a full factorial DoSs, parameter estimation method can be used to work out the empirical equations. For a sandpile example, repose angle or FR can be expressed as $\alpha$ or $\theta$ or FR $=A \cdot \varepsilon_{p}^{b}$. $\mu_{s, p p}^{c} \cdot \mu_{r, p p}^{d}$.

- Level 2 parameter sensitivity coefficients were derived from fundamental equations applied in DEM simulations (e.g., Hertz-Mindlin). A parameter sensitivity matrix was constructed and linked to the bulk observations from the DoSs. PCA is used to find the weight of the contribution from each parameter, which is implied by the eigenvectors of the sensitivity matrix.

- Level 3 parameter impact sensitivity on the intrinsic physical model response (e.g., contact force and torque) can be analysed, again using the parameter sensitivity matrix. Cross-correlation coefficients between parameters can be obtained from the covariance matrix of the parameter sensitivity matrix. In this approach, a priori checks can be made before running simulations to indicate which parameters should be best understood. This provides better preliminary indications of which parameters should be measured and where the calibration or material properties measurements effort should be focused. This has the potential to reduce computation time and avoid producing misleading results from simulations.

Acknowledgments This work was supported by the IPROCOM Marie Curie Initial Training Network, funded through the People Programme (Marie Curie Actions) of the European Union's Seventh Framework Programme FP7/2007-2013/ under REA Grant Agreement No. 316555.

\section{References}

1. Cundall PA, Strack ODL (1979) A discrete numerical model for granular assemblies. Geotechnique 29(1):47-65

2. Matuttis HG, Luding S, Herrmann HJ (2000) Discrete element simulations of dense packings and heaps made of spherical and non-spherical particles. Powder Technol 109(1-3):278-292

3. Zhang ZP, Liu LF, Yuan YD, Yu AB (2001) A simulation study of the effects of dynamic variables on the packing of spheres. Powder Technol 116(3):23-32 
4. Dutt M, Hancock BC, Bentham AC, Elliott JA (2005) An implementation of granular dynamics for simulating frictional elastic particles based on the DL-POLY code. Comput Phys Commun 166(1):26-44

5. Cleary PW, Sawley ML (2003) DEM modelling of industrial granular flows: 3D case studies and the effect of particle shape on hopper discharge. Appl Math Model 26(2):89-111

6. Ketterhagen WR, Curtis JS, Wassgren CR, Hancock BC (2009) Predicting the flow mode from hoppers using the discrete element method. Powder Technol 195(1):1-10

7. Anand A, Curtis JS, Wassgren CR, Hancock BC, Ketterhagen WR (2008) Predicting discharge dynamics from a rectangular hopper using the discrete element method (DEM). Chem Eng Sci 63(24):5821-5830

8. Wu CY (2008) DEM simulations of die filling during pharmaceutical tableting. Particuology 6(6):412-418

9. Guo Y, Wu CY, Kafui KD, Thornton C (2010) Numerical analysis of density-induced segregation during die filling. Powder Technol 197(1-2):111-119

10. Thornton C, Yin KK, Adams MJ (1996) Numerical simulation of the impact fracture and fragmentation of agglomerates. J Phys D 29(2):424-435

11. Liu L, Kafui KD, Thornton C (2010) Impact breakage of spherical, cuboidal and cylindrical agglomerates. Powder Technol 199(2):89196

12. Martin CL, Bouvard D (2003) Study of the cold compaction of composite powders by the discrete element method. Acta Mater 51(2):373-386

13. Samimi A, Hassanpour A, Ghadiri M (2005) Single and bulk compressions of soft granules: experimental study and DEM evaluation. Chem Eng Sci 60(14):3993-4004

14. Hassanpour A, Ghadiri M (2004) Distinct element analysis and experimental evaluation of the Heckel analysis of bulk powder compression. Powder Technol 141(3):251-261

15. Markauskas D, Kacianauskas R (2006) Compacting of particles for biaxial compression test by discrete element method. J Civ Eng Manag 12(2):153-161

16. Moysey PA, Thompson MR (2005) Modelling the solids inflow and solids conveying of single-screw extruders using the discrete element method. Powder Technol 153(2):95-107

17. Owen PJ, Cleary PW (2009) Prediction of screw conveyor performance using the Discrete Element Method (DEM). Powder Technol 193(3):274-288

18. Arratia PE, Duong N, Muzzio FJ, Godbole P, Reynolds S (2006) A study of the mixing and segregation mechanisms in the Bohle Tote blender via DEM simulations. Powder Technol 164(1):50-57

19. Yang RY, Yu AB, McElroy L, Bao J (2008) Numerical simulation of particle dynamics in different flow regimes in a rotating drum. Powder Technol 188(2):170-177

20. Marigo M, Cairns DL, Davies M, Cook M, Ingram A, Stitt EH (2010) Developing mechanistic understanding of granular behaviour in complex moving geometry using the discrete element method. Part A: measurement and reconstruction of Turbula mixer motion using positron emission particle tracking. Comput Model Eng Sci 59(3):217-238

21. Alizadeh E, Bertrand F, Chaouki J (2014) Discrete element simulation of particle mixing and segregation in a tetrapodal blender. Comput Chem Eng 64:1-12

22. Leisena D, Kerkamma I, Bohna E, Kamlah M (2012) A novel and simple approach for characterizing the Young's modulus of single particles in a soft matrix by nanoindentation. J Mater Res 27(24):3073-3082

23. Marigo M, Cairns DL, Bowen J, Ingram A, Stitt EH (2014) Relationship between single and bulk mechanical properties for zeolite ZSM5 spray-dried particles. Particuology 14:130-138
24. Prabhu B (2005) Microstructural and mechanical characterization of $\mathrm{Al}-\mathrm{Al}_{2} \mathrm{O}_{3}$ nanocomposites synthesized by high-energy milling. Dissertation, University of Central Florida, Orlando

25. Davies M, Brindley A, Chen X, Marlow M, Doughty SW, Shrubb I, Roberts CJ (2005) Characterization of drug particle surface energetics and Young's modulus by atomic force microscopy and inverse gas chromatography. Pharm Res 22(7):1158-1166

26. Jones R (2003) From single particle AFM studies of adhesion and friction to bulk flow: forging the links. Granul Matter 4(4):191-204

27. Marigo M, Stitt EH (2014) Discrete element method (DEM) for industrial applications: comments on calibration and validation for the modelling of cylindrical pellets. KONA Powder Part J 32:236252

28. Dong H, Moys MH (2003) Measurement of impact behaviour between balls and walls in grinding mills. Miner Eng 16(6):543550

29. Stitt EH, Marigo M, Wilkinson SK, Dixon AG (2015) How good is your model? Johns Matthey Technol Rev 59(2):74-89

30. Sjöblom J (2009) Parameter estimation in heterogeneous catalysis. Dissertation, Chalmers University of Technology

31. Zhou YC, Xu BH, Yu AB, Zulli P (2002) An experimental and numerical study of the angle of repose of coarse spheres. Powder Technol 125(1):45-54

32. Wilkinson SK (2014) Reaction kinetics in formulated industrial catalysts. EngD Thesis, University of Birmingham

33. Sjöblom J, Creaser D (2007) New approach for microkinetic mean-field modelling using latent variables. Comput Chem Eng 31(4):307-317

34. Jansson J (2002) Studies of catalytic low-temperature CO oxidation over cobalt oxide and related transition metal oxides. Dissertation, Chalmers University of Technology

35. Quiney AS, Schuurman Y (2007) Kinetic modelling of CO conversion over a Cu/ceria catalyst. Chem Eng Sci 62(18-20):5026-5032

36. Aarts R, Irwan R, Janssen AJEM (2002) Efficient tracking of the cross correlation coefficient. IEEE Trans Speech Audio Process 10(6):391-402

37. Vadja S, Valko P, Turanyi T (1985) Principal component analysis of kinetic model. Int J Chem Kinet 17(1):55-81

38. Mhadeshwar AB, Vlachos DG (2005) Is the water-gas shift reaction on Pt simple?: Computer-aided microkinetic model reduction, lumped rate expression, and rate-determining step. Catal Today 105(1):162-172

39. Raimondeau S, Aghalayam P, Mhadeshwar AB, Vlachos DG (2003) Parameter optimization of molecular models: application to surface kinetics. Ind Eng Chem Res 42(6):1174-1183

40. Rogers A, Ierapetritou MG (2014) Discrete element reduced-order modelling of dynamic particulate systems. AIChE J 60(9):31843194

41. Hertz H (1881) Ueber die Berührung fester elastischer Koerper. J Reine Angew Math 92:156-171

42. Mindlin RD (1949) Compliance of elastic bodies in contact. ASME Trans J Appl Mech 16:259-268

43. Ai J, Chen JF, Rotter JM, Ooi JY (2011) Assessment of rolling resistance models in discrete element simulations. Powder Technol 206(3):269-282

44. Thornton C, Randall CW (1988) Applications of theoretical contact mechanics to solid particle system simulation. In: Satake M, Jenkins TJ (eds) Micromechanics Granular matters. Elsevier, Amsterdam, pp 133-142

45. Vajda S, Valko P, Turanyi T (1985) Principal component analysis of kinetic models. Int J Chem Kinet 17(1):55-81

46. Marquardt DW (1963) An algorithm for least-squares estimation of nonlinear parameters. J Soc Ind Appl Math 11(2):431-441

47. Kloss C, Goniva C, Hager A, Amberger S, Pirker S (2012) Models, algorithms and validation for open source DEM and CFD-DEM. Int J Prog Comput Fluid Dyn 12(2):140-152 
48. Zhu HP, Yu AB (2005) Steady-state granular flow in a 3D cylindrical hopper with fat bottom: macroscopic analysis. Granul Matter 7:97-107

49. Liu SD, Zhou ZY, Zou RP, Pinson D, Yu AB (2014) Flow characteristics and discharge rate of ellipsoidal particles in a flat bottom hopper. Powder Technol 253:70-79

50. Ooi JY (2013) Establishing predictive capabilities of DEMverification and validation for complex granular processes. Powders Grains 2013 Proc 7th Int Conf Micromech Granul Media 1542(1):20-24

51. Persson AS, Alderborn G, Frenning G (2011) Flowability of surface modified pharmaceutical granules: a comparative experimental and numerical study. Eur J Pharm Sci 42(3):199-209
52. Li Y, Xu Y, Thornton C (2005) A comparison of discrete element simulations and experiments for 'sandpiles' composed of spherical particles. Powder Technol 160(3):219-228

53. Zhao J, Shan T (2013) Numerical modeling of fluid-particle interaction in granular media. Theor Appl Mech Lett 3(2):021007

54. Lommen S, Schott D, Lodewijks G (2014) DEM speedup: stiffness effects on behavior of bulk material. Particuology 12:107-112

55. Moon SJ, Swift JB, Swinney HL (2004) Role of friction in pattern formation in oscillated granular layers. Phys Rev E 69(3):031301 